\title{
Screening for characteristic microRNAs between pre-invasive and invasive stages of cervical cancer
}

\author{
XIAO-LU ZHU, SHANG-YUN WEN, ZHI-HONG AI, JUAN WANG, YAN-LI XU and YIN-CHENG TENG \\ Department of Obstetrics and Gynecology, Shanghai Sixth People's Hospital, \\ Shanghai Jiaotong University, Shanghai 200233, P.R. China
}

Received March 13, 2014; Accepted December 12, 2014

DOI: $10.3892 / \mathrm{mmr} .2015 .3363$

\begin{abstract}
The aim of the present study was to investigate the characteristic microRNAs (miRNAs) expressed during the pre-invasive and invasive stages of cervical cancer. A gene expression profile (GSE7803) containing 21 invasive squamous cell cervical carcinoma samples, 10 normal squamous cervical epithelium samples and seven high-grade squamous intraepithelial cervical lesion samples, was obtained from the Gene Expression Omnibus. Differentially expressed genes (DEGs) were identified using significance analysis of microarray software, and a Gene Ontology (GO) enrichment analysis was conducted using the Database for Annotation, Visualization and Integrated Discovery. The miRNAs that interacted with the identified DEGs were selected, based on the TarBase v5.0 database. Regulatory networks were constructed from these selected miRNAs along with their corresponding target genes among the DEGs. The regulatory networks were visualized using Cytoscape. A total of 1,160 and 756 DEGs were identified in the pre-invasive and invasive stages of cervical cancer, respectively. The results of the GO enrichment demonstrated that the DEGs were predominantly involved in the immune response and the cell cycle, in the pre-invasive and invasive stages, respectively. Furthermore, a total of 18 and 26 characteristic miRNAs were screened in the pre-invasive and invasive stages, respectively. These miRNAs may be potential biomarkers and targets for the diagnosis and treatment of the different stages of cervical cancer.
\end{abstract}

\section{Introduction}

Cervical cancer is the most common gynecological malignancy, and is the second leading cause of cancer-associated mortality in females worldwide $(1,2)$. One reason for the high

Correspondence to: Dr Yin-Cheng Teng, Department of Obstetrics and Gynecology, Shanghai Sixth People's Hospital, Shanghai Jiaotong University, 600 Yishan Road, Shanghai 200233, P.R. China E-mail: tengyinchengij@hotmail.com

Key words: cervical cancer, invasion, differentially expressed gene, gene ontology, microRNA-target gene network levels of prevalence of this cancer is the lack of awareness and early detection approaches $(3,4)$. Therefore, understanding the underlying molecular mechanisms of cervical cancer, and establishing more effective therapies are important areas of ongoing research.

The identification and characterization of key microRNAs (miRNAs) that participate in cervical cancer, is essential for determining the underlying mechanisms of this disease and establishing novel therapeutic strategies. miRNAs are 20-24 nt RNAs that are derived from distinct hairpin precursors in animals, plants and fungi, which bind to complementary sequences on target mRNAs $(5,6)$. miRNAs regulate gene expression by cleaving target mRNAs, and by translational suppression at the post-transcriptional level (7). Previous studies have shown that miRNAs have important roles in various biological and metabolic processes, including cell growth, apoptosis, viral infection, differentiation, signal transduction and cancer (8-11). Numerous studies have demonstrated that miRNAs are involved in the initiation and progression of cancer, and may be potential biomarkers for the diagnosis and prognosis of tumors, in addition to functioning as potential therapeutic targets (12-14). Therefore, it may be beneficial to identify novel miRNAs to act as diagnostic and therapeutic biomarkers, or therapeutic targets, in cervical cancer.

Recently, molecular network analysis technology, combined with gene expression profile data, has exhibited potential in a number of areas, including classification of diseases and the identification of novel therapeutic targets $(15,16)$. In the present study a microarray dataset of healthy and malignant cervical samples was downloaded from the Gene Expression Omnibus (GEO) database. Differentially expressed genes (DEGs) were identified between these groups. Based on the TarBase v5.0 database, regulatory networks were constructed from selected miRNAs and their corresponding target genes from the identified DEGs. Key miRNAs, which may be used as potential biomarkers or therapeutic targets in cervical cancer, were subsequently identified.

\section{Materials and methods}

Affymetrix microarray data. A gene expression profile generated by Zhai et al (17) was used in the present study, which was deposited in the GEO database (http://www.ncbi.nlm.nih. gov/geo/query/acc.cgi?acc=GSE7803). This gene expression 
profile is based on the GPL96 platform (Affymetrix Human Genome U133A Array). A total of 38 samples were available, including 21 invasive squamous cell cervical carcinoma (SCC) samples, ten normal squamous cervical epithelium (NE) samples and seven high-grade squamous intraepithelial cervical lesion (HSIL) samples.

Screening of DEGs. In order to identify the DEGs, the original GSE7803 dataset was converted into an identifiable expression form and was normalized. Probe sets were mapped to the National Centers of Biotechnology Information genes (http://www.ncbi.nlm.nih.gov). Probe sets that corresponded to numerous genes or to no genes were removed from subsequent analyses. For genes that corresponded with numerous probe sets and had a plurality of expression values, the expression values were averaged. Subsequently, the SAMR package (18) in R and a significance analysis of microarray (SAM) were used to identify the DEGs between the samples (19). SAM software is a practical tool used for detecting significantly expressed genes, and for controlling the proportion of falsely detected genes. In the present study, genes with a fold-change $>1.2$ and a false discovery rate (FDR) $<0.05$ were selected as DEGs. In addition, the identified DEGs were divided into two groups: DEGs from the NE and HSIL samples were considered pre-invasive DEGs, whereas DEGs from the HSIL and invasive SCC samples were considered invasive DEGs.

Functional enrichment analysis of DEGs. The Database for Annotation, Visualization and Integrated Discovery (DAVID; http://david.abcc.ncifcrf.gov/) is a web-accessible program that provides a comprehensive set of functional annotation tools, which may be used by investigators to understand the underlying biological functions of large lists of genes (20). The present study used DAVID to perform a Gene Ontology (GO) enrichment analysis of the identified DEGs. Based on hypergeometric distribution, GO terms were enriched, and numerous testing corrections were conducted using the Benjamini-Hochberg method (21). An FDR $<0.05$ was set as the cut-off value.

Construction of regulatory networks. TarBase is a database that contains a manually curated collection of experimentally supported miRNA targets from a animal, pant and viral species of central scientific interest (22). TarBase v5.0 is the updated and extended version of the TarBase database, with $>1,300$ experimentally supported miRNA-target interactions (MTIs). It contains 1,094 human MTIs between 285 miRNAs and 1,721 target genes.

In the present study, human miRNA target gene data were downloaded from the TarBase v5.0 database (http://diana. cslab.ece.ntua.gr/tarbase/). miRNAs that interacted with the identified DEGs were then selected. Subsequently, MTIs regulatory networks were constructed from these selected miRNAs and their corresponding target genes within the DEGs. The MTIs regulatory networks were visualized by Cytoscape (23). In addition, the MTIs regulatory networks were divided into two groups: The regulatory network constructed from the selected miRNAs and the pre-invasive DEGs was termed the pre-invasive regulatory network, whereas the regulatory network constructed from the selected miRNAs and the invasive DEGs was termed the invasive regulatory network.

Comparison of the regulatory networks. In order to determine the differences between the pre-invasive and invasive stages of cervical cancer, regulatory networks were constructed and compared. Regulatory networks may be characterized by topological properties, such as degree (24). Degree is defined as the number of edges per node, which indicates the number of interacting partners. The present study used Freeman's degree centrality to analyze the degree of the regulatory networks (25). Freeman's degree centrality consists of ingoing (in-degree) and outgoing degree (out-degree). In-degree refers to the number of links a node receives from other nodes, whereas out-degree refers to the number of links originating from a particular node.

\section{Results}

DEG analysis. The original GSE7803 dataset was downloaded from the GEO database, and the DEGs were identified using SAM. Genes with a fold-change $>1.2$ and an FDR $<0.05$ were classed as DEGs. A total of 1,160 pre-invasive and 756 invasive DEGs were identified. In addition, 2,001 DEGs were identified from the NE and invasive SCC samples.

GO analysis of DEGs. In order to study the DEGs that contributed to cervical cancer, a GO enrichment analysis for the pre-invasive and invasive DEGs was performed using DAVID software. The pre-invasive DEGs (e.g. PSMB10, POU2AF1, ST6GAL1, CLU, SERPING1 and APOL2) were predominantly involved in the immune response, such as the acute inflammatory response (FDR=3.06E-04; Table I). By contrast, the invasive DEGs (e.g. TTK, AURKA, BRCA2, PSMC3IP, CDK10 and TUBG1) were predominantly involved in the regulation of the cell cycle, such as Cell Cycle (FDR=1.25E-19; Table II).

Construction of regulatory networks. Based on human MTIs data, pre-invasive and invasive regulatory networks were constructed. The pre-invasive regulatory network consisted of 80 pairs of regulatory interactions between 18 miRNAs and 66 pre-invasive DEGs (Fig. 1). The invasive regulatory network consisted of 64 pairs of regulatory interactions between 26 miRNAs and 51 invasive DEGs (Fig. 2). The highest out-degree was observed in miR-124, in the pre-invasive as well as the invasive regulatory networks.

Comparisons between the regulatory networks. Based on the topological properties of the networks, the similarities and differences between the pre-invasive and invasive regulatory networks were identified. The invasive regulatory network (Fig. 2) consisted of many smaller sub-networks and the out-degree of miRNAs was decreased, compared with those in the pre-invasive regulatory network (Fig. 1). For example, there were 14 DEGs associated with miR-1, and 21 DEGs associated with miR-124 in the pre-invasive regulatory network (Fig. 1). However, only eight and nine DEGs were associated with miR-1 and miR-124 in the invasive regulatory network, respectively (Fig. 2). 


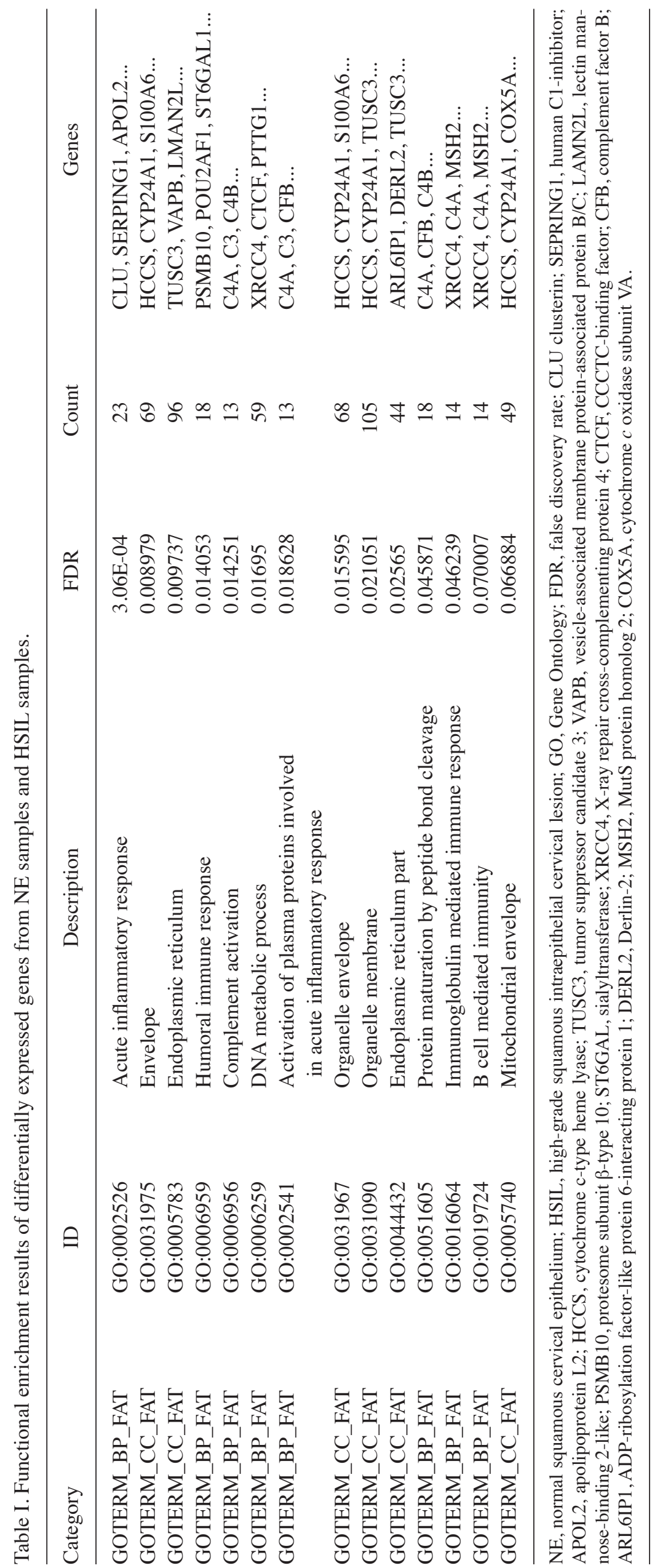




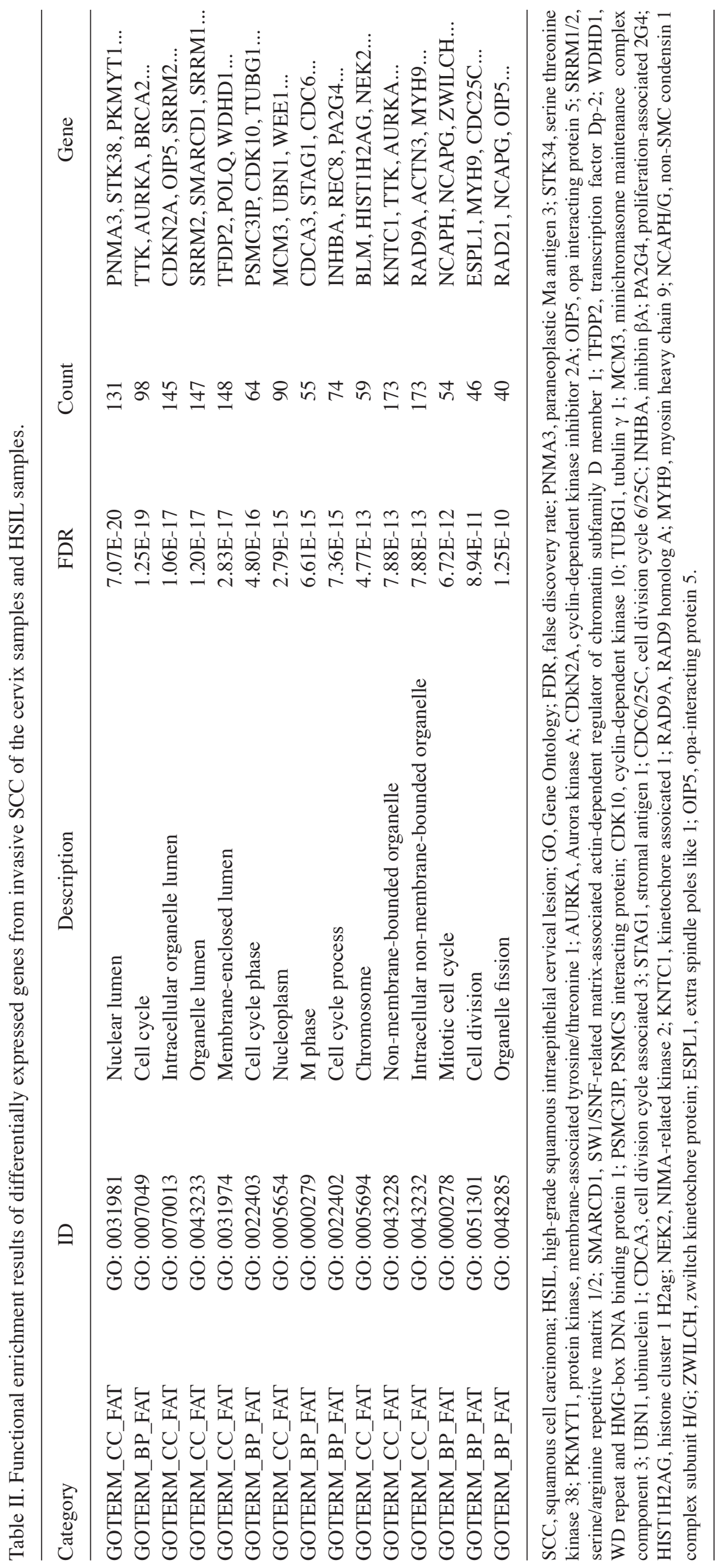




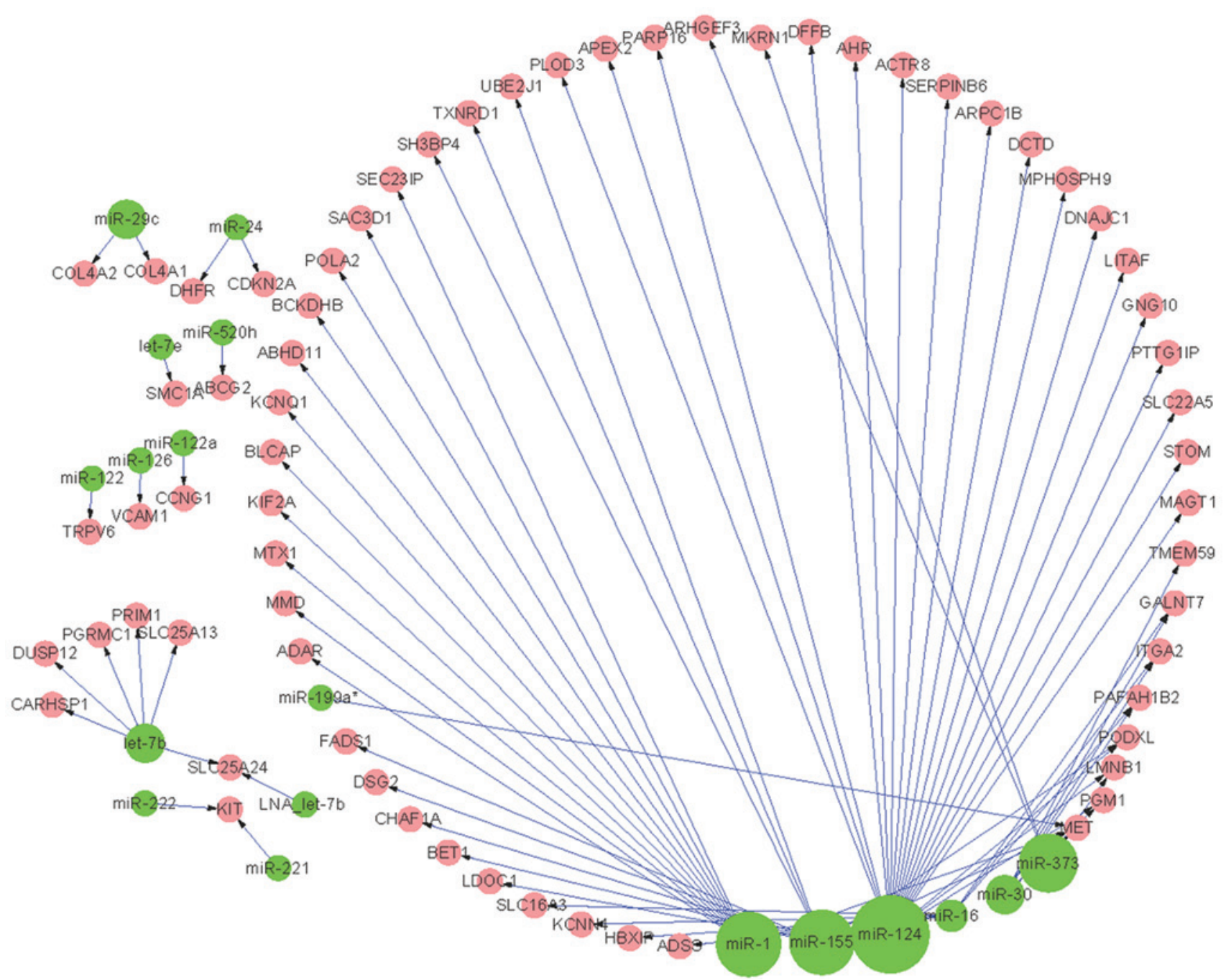

Figure 1. Pre-invasive regulatory network. Regulatory network constructed by microRNAs and differentially expressed genes from normal squamous cervical epitheilum and high grade squamous intraepithelial cervical lesion samples. Red nodes represent target differentially expressed genes and green nodes represent microRNAs. Blue lines represent microRNA-target regulatory interactions (in-degree), and arrows indicate microRNA target differentially expressed genes (out-degree). The size of each green node represents the out-degree. As the out-degree increases, the associated green node becomes larger.

A total of 10 common miRNAs were identified in the regulatory networks. Three miRNAs: miR-1, miR-124 and miR-16, had a degree change $>5$. In addition, there were eight miRNAs that were only detected in the pre-invasive regulatory network (Fig. 1), including miR-126 and miR-199a. By contrast, there were 16 miRNAs that were only detected in the invasive regulatory network (Fig. 2), including miR-127, miR-143, miR-17-5p, miR-26a, miR-29a, miR-34a and miR-375.

\section{Discussion}

Malignant transformation during tumor progression results from a series of genetic alterations (26). In order to gain a better understanding of the genetic changes that occur during the progression of cervical cancer, a gene expression profile (GSE7803) was analyzed using a bioinformatics approach. In the present study, a total of 756 invasive DEGs, 1,160 pre-invasive DEGs, and 2,001 DEGs from invasive SCC and NE samples, were identified. These findings are in accordance with those of previous studies, which have consistently shown that the expression of genes is markedly altered in invasive tumor cells, compared with that of noninvasive and normal cells $(27,28)$. Furthermore, the results of a GO enrichment of the identified DEGs, indicated that the expression of key genes differs between the pre-invasive and invasive stages of cervical cancer.

Clusterin (CLU) was initially identified as a secreted glycoprotein that has a cytoprotective role. However, numerous intracellular CLU variants have recently been identified in diverse pathological conditions (29-31). Furthermore, recent studies have shown that CLU is involved in various biological functions, such as cell death, tumor progression and neurodegenerative disorders $(32,33)$. A previous study used DNA microarray data to identify novel candidate molecular markers for cervical cancer diagnosis and therapy, and observed the downregulation of human $\mathrm{C} 1$ inhibitor (SERPING1) in invasive cervical carcinoma cells (34). In addition, a recent genomic study demonstrated that apolipoprotein L2 (APOL2) is markedly upregulated in cervical cancer (35). These findings, as well as the results of the present study, indicate that CLU, SERPING1 and APOL2 may have important roles in the progression of cervical cancer. 


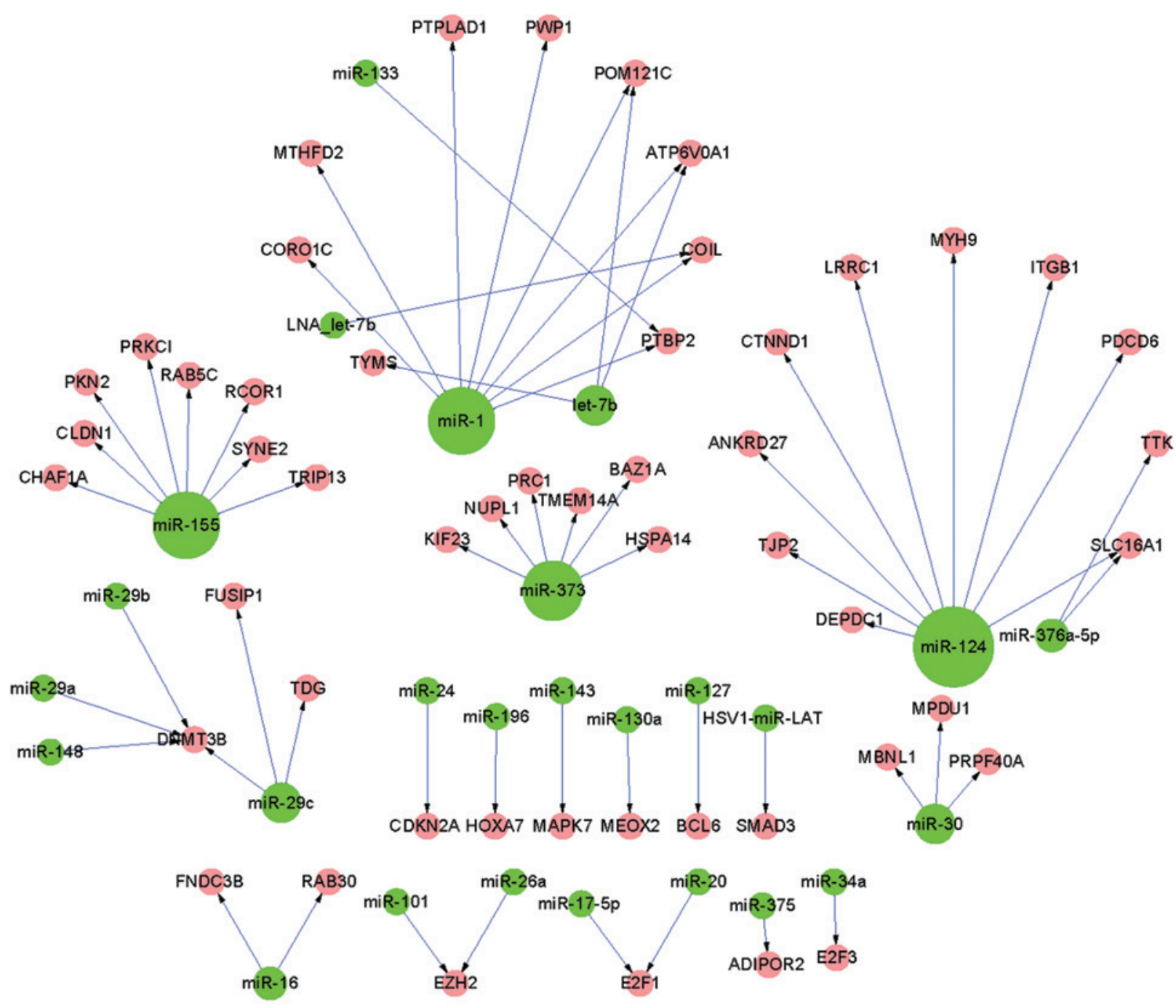

Figure 2. Invasive regulatory network. Regulatory network constructed by microRNAs and differentially expressed genes from invasive squamous cell cervical carcinoma samples and high grade squamous intraepithelial cervical lesion samples. Red nodes represent target differentially expressed genes and green nodes represent microRNAs. Blue lines represent microRNA-target regulatory interactions (in-degree), and arrows indicate microRNA target differentially expressed genes (out-degree). The size of each green node represents the out-degree. As the out-degree increases, the green node becomes bigger.

TTK has been shown to be associated with metastasis via chromosomal instability, in a previous study, which aimed to identify genes associated with the progression and metastasis of advanced cervical cancer following radiotherapy (36). Furthermore, genetic variants of Aurora A kinase (AURKA) have been shown to be associated with a radiotherapy-induced early adverse reaction in patients with cervical cancer (37). Previous studies have demonstrated that both BRCA1 and BRCA2 participate in a common DNA damage response pathway, and are involved in the activation of homologous recombination and double-strand break repair (38). By contrast, Narayan et al (39) reported the downregulation of BRCA1 in a small subset of patients with cervical cancer. These previous findings and the results of the present analysis suggest that TTK, AURKA and BRCA2 may participate in the progression of cervical cancer.

In order to obtain the upstream regulatory information of the DEGs, two regulatory networks were constructed based on the TarBase v5.0 database. These regulatory networks were then compared, and the common and specific miRNAs were identified. The miRNA with the highest out-degree was shown to be miR-124, in the pre-invasive as well as the invasive regulatory networks. miR-124 has previously been shown to be the most abundant miRNA expressed in neuronal cells (40). Furthermore, previous studies have shown that the upregulation of miR-124 induces neuronal differentiation of various tumor cell lines in mice (41-43). Wilting et al (44) previously demonstrated that the silencing of miR-124 expression, by methylation, inhibited the development of cervical carcinoma. These results suggest that miR-124 may be a potential therapeutic target for cervical cancer therapy.

Of the eight miRNAs specific to the pre-invasive regulatory network, miR-126 has previously been reported to be downregulated in cervical cancer tissues (45), and miR-199a has previously been suggested as a potential therapeutic target for cervical cancer therapy (46). miR-126 is a human miRNA that is expressed only in endothelial cells, throughout capillaries as well as in larger blood vessels (47), and acts upon 
various transcripts in order to control angiogenesis (48). miR-126 has been identified as a tumor suppressor and as an oncogene, depending on the type of cancer involved. Inhibition of cancer progression by miR-126 is achieved through the negative control of proliferation, migration, invasion and cell survival. However, miR-126 may also support cancer progression through the promotion of blood vessel formation and inflammation at the site of activation (49). According to these previous findings and the results of the present study, miR-126 may be a potential biomarker for the diagnosis of cervical cancer, and a therapeutic target for the pre-invasive stage of this disease.

Of the 16 miRNAs specific to the invasive regulatory network, seven have been reported in previous studies and described as being upregulated or downregulated in cervical cancer. These include miR-127, miR-143, miR-17-5p, miR-26a, miR-29a, miR-34a and miR-375 (45,50-54). Lee et al (46) demonstrated that the expression of miR-127 was significantly increased in patients with invasive squamous cell carcinoma, which had metastasized to the lymph nodes. The results of the present study are in accordance with those of previous studies, which indicate that miR-127 may be a marker for lymph node metastasis in invasive cervical cancer. miR-143 is highly conserved in vertebrates (55) and changes in miR-143 expression have frequently been implicated in cancer (56-58). Furthermore, the upregulation of miR-143 has previously been observed in a hepatocellular carcinoma model during tumor metastasis, through repression of FNDC38 (59). However, reduced expression of miR-143 has also been observed in a range of cancer stages, including at very early stages (60). The results of previous studies and of the present study indicate that miR-143 may be involved in tumor progression, and may be a candidate for RNA-targeted treatment of tumors (61). Wang et al (52) previously reported that miR-375 is downregulated in squamous cervical cancer, and inhibits cell migration and invasion by targeting the transcription factor, SP1. This finding indicates that deregulation of miR-375 may have an important role in the malignant transformation of cervical cancer cells. However, the elucidation of the underlying molecular mechanisms of miR-17-5p, miR-26a, miR-29a and miR-34a in the progression of cervical cancer, and the use of other miRNAs screened in the present study as biomarkers or therapeutic targets in cervical cancer require further investigation.

In conclusion, a total of 1,160 and 756 DEGs were identified in the pre-invasive and invasive stages of cervical cancer, respectively. The GO enrichment analysis demonstrated that the DEGs were primarily involved in the immune response and regulation of the cell cycle, in the pre-invasive and invasive stages, respectively. These findings indicate that the expression of key genes differs between the pre-invasive and invasive stages of cervical cancer progression. Based on the analysis of the regulatory networks, a total of 18 and 26 key miRNAs were screened in the pre-invasive and invasive stages, respectively. It is hypothesized that these miRNAs are involved in the malignant transformation of cervical cancer cells. In addition, these miRNAs may have a function as novel biomarkers in cervical cancer diagnosis and detection, and as therapeutic targets in this disease. Further studies in independent patient cohorts are required, in order to validate the potential roles of these miRNAs.

\section{References}

1. Jemal A, Bray F, Center MM, Ferlay J, Ward E and Forman D: Global cancer statistics. CA Cancer J Clin 61: 69-90, 2011.

2. Parkin DM, Bray F, Ferlay J and Pisani P: Estimating the world cancer burden: Globocan 2000. Int J Cancer 94: 153-156, 2001.

3. Colomiere M, Ward AC, Riley C, et al: Cross talk of signals between EGFR and IL-6R through JAK2/STAT3 mediate epithelial-mesenchymal transition in ovarian carcinomas. Br J Cancer 100: 134-144, 2009.

4. Ono K, Tanaka T, Tsunoda T, et al: Identification by cDNA microarray of genes involved in ovarian carcinogenesis. Cancer Res 60: 5007-5011, 2000.

5. Rhoades MW, Reinhart BJ, Lim LP, Burge CB, Bartel B and Bartel DP: Prediction of plant microRNA targets. Cell 110: 513-520, 2002

6. Lu Y, Zhou Y, Qu W, Deng M and Zhang C: A Lasso regression model for the construction of microRNA-target regulatory networks. Bioinformatics 27: 2406-2413, 2011.

7. Ying SY, Chang DC and Lin SL: The microRNA (miRNA): Overview of the RNA genes that modulate gene function. Mol Biotech 38: 257-268, 2008.

8. Pritchard CC, Kroh E, Wood B, et al: Blood cell origin of circulating microRNAs: a cautionary note for cancer biomarker studies. Cancer Prev Res (Phila) 5: 492-497, 2012.

9. Farazi TA, Hoell JI, Morozov P and Tuschl T: MicroRNAs in human cancer. Adv Exp Med Biol 774: 1-20, 2013.

10. Balch C, Naegeli K, Nam S, et al: A unique histone deacetylase inhibitor alters microRNA expression and signal transduction in chemoresistant ovarian cancer cells. Cancer Biol Ther 13: 681-693, 2012.

11. Aydoğdu E, Katchy A, Tsouko E, et al: MicroRNA-regulated gene networks during mammary cell differentiation are associated with breast cancer. Carcinogenesis 33: 1502-1511, 2012.

12. Lu J, Getz G, Miska EA, et al: MicroRNA expression profiles classify human cancers. Nature 435: 834-838, 2005.

13. Garzon R, Fabbri M, Cimmino A, Calin GA and Croce CM: MicroRNA expression and function in cancer. Trends Mol Med 12: 580-587, 2006.

14. Cho WC: MicroRNAs: potential biomarkers for cancer diagnosis, prognosis and targets for therapy. Int J Biochem Cell Biol 42: 1273-1281, 2010.

15. Wu SF, Qian WY, Zhang JW, et al: Network motifs in the transcriptional regulation network of cervical carcinoma cells respond to EGF. Arch Gynecol Obstet 287: 771-777, 2013.

16. Higareda-Almaraz JC, Enríquez-Gasca Mdel R, Hernández-Ortiz M, Resendis-Antonio $O$ and Encarnación-Guevara S: Proteomic patterns of cervical cancer cell lines, a network perspective. BMC Sys Biol 5: 96, 2011.

17. Zhai Y, Kuick R, Nan B, et al: Gene expression analysis of preinvasive and invasive cervical squamous cell carcinomas identifies HOXC10 as a key mediator of invasion. Cancer Res 67: 10163-10172, 2007.

18. Tusher VG, Tibshirani R and Chu G: Significance analysis of microarrays applied to the ionizing radiation response. Proc Natl Acad Sci USA 98: 5116-5121, 2001.

19. Larsson O, Wahlestedt $C$ and Timmons JA: Considerations when using the significance analysis of microarrays (SAM) algorithm. BMC Bioinformatics 6: 129, 2005.

20. Dennis G Jr, Sherman BT, Hosack DA, et al: DAVID: Database for annotation, visualization and integrated discovery. Genome Biol 4: P3, 2003.

21. Benjamini Y and Hochberg Y: Controlling the false discovery rate: A practical and powerful approach to multiple testing. J R Stat Soc Series B Stat Methodol 57: 289-300, 1995.

22. Papadopoulos GL, Reczko M, Simossis VA, Sethupathy P and Hatzigeorgiou AG: The database of experimentally supported targets: a functional update of TarBase. Nucleic Acids Res 37: D155-D158, 2009.

23. Shannon P, Markiel A, Ozier O, et al: Cytoscape: a software environment for integrated models of biomolecular interaction networks. Genome Res 13: 2498-2504, 2003.

24. Zhang S, Jin G, Zhang XS and Chen L: Discovering functions and revealing mechanisms at molecular level from biological networks. Proteomics 7: 2856-2869, 2007.

25. Freeman LC: Centrality in social networks conceptual clarification. Soc Networks 1: 215-239, 1979.

26. Fearon ER and Vogelstein B: A genetic model for colorectal tumorigenesis. Cell 61: 759-767, 1990. 
27. Ryu B, Jones J, Hollingsworth MA, Hruban RH and Kern SE: Invasion-specific genes in malignancy: serial analysis of gene expression comparisons of primary and passaged cancers. Cancer Res 61: 1833-1838, 2001.

28. Kitahara O, Furukawa Y, Tanaka T, et al: Alterations of gene expression during colorectal carcinogenesis revealed by cDNA microarrays after laser-capture microdissection of tumor tissues and normal epithelia. Cancer Res 61: 3544-3549, 2001.

29. Reddy KB, Jin G, Karode MC, Harmony JA and Howe PH: Transforming growth factor beta (TGF beta)-induced nuclear localization of apolipoprotein $\mathrm{J} /$ clusterin in epithelial cells. Biochemistry 35: 6157-6163, 1996.

30. Yang CR, Leskov K, Hosley-Eberlein K, et al: Nuclear clusterin/XIP8, an x-ray-induced Ku70-binding protein that signals cell death. Proc Natl Acad Sci USA 97: 5907-5912, 2000

31. O'Sullivan J, Whyte L, Drake J and Tenniswood M: Alterations in the post-translational modification and intracellular trafficking of clusterin in MCF-7 cells during apoptosis. Cell Death Differ 10: 914-927, 2003.

32. Klokov D, Leskov K, Araki S, et al: Low dose IR-induced IGF-1-sCLU expression: a p53-repressed expression cascade that interferes with TGFbeta1 signaling to confer a pro-survival bystander effect. Oncogene 32: 479-490, 2013.

33. Choi I, Kim J, Park JY and Kang SW: Cotransin induces accumulation of a cytotoxic clusterin variant that cotranslationally rerouted to the cytosol. Exp Cell Res 319: 1073-1082, 2013.

34. Santin AD, Zhan F, Bignotti E, et al: Gene expression profiles of primary HPV16- and HPV18-infected early stage cervical cancers and normal cervical epithelium: identification of novel candidate molecular markers for cervical cancer diagnosis and therapy. Virology 331: 269-291, 2005.

35. Ahn WS, Bae SM, Lee JM, et al: Searching for pathogenic gene functions to cervical cancer. Gynecol Oncol 93: 41-48, 2004

36. Harima Y, Ikeda K, Utsunomiya K, et al: Identification of genes associated with progression and metastasis of advanced cervical cancers after radiotherapy by cDNA microarray analysis. Int J Radiat Oncol 75: 1232-1239, 2009.

37. Ishikawa A, Suga T, Shoji Y, et al: Genetic Variants of NPAT-ATM and AURKA are associated with an early adverse reaction in the gastrointestinal tract of patients with cervical cancer treated with pelvic radiation therapy. Int J Radiat Oncol 81: 1144-1152, 2011.

38. Chen JJ, Silver D, Cantor S, Livingston DM and Scully R: BRCA1, BRCA2 and Rad51 operate in a common DNA damage response pathway. Cancer Res 59 Suppl 7: 1752-1756, 1999.

39. Narayan G, Arias-Pulido H, Nandula SV, et al: Promoter hypermethylation of FANCF disruption of Fanconi Anemia-BRCA pathway in cervical cancer. Cancer Res 64: 2994-2997, 2004

40. Lagos-Quintana M, Rauhut R, Meyer J, Borkhardt A and Tuschl T: New microRNAs from mouse and human. RNA 9: 175-179, 2003

41. Makeyev EV, Zhang J, Carrasco MA and Maniatis T: The MicroRNA miR-124 promotes neuronal differentiation by triggering brain-specific alternative pre-mRNA splicing. Mol Cell 27: 435-448, 2007.

42. Smirnova L, Gräfe A, Seiler A, Schumacher S, Nitsch R and Wulczyn FG: Regulation of miRNA expression during neural cell specification. Eur J Neurosci 21: 1469-1477, 2005.

43. Krichevsky AM, King KS, Donahue CP, Khrapko K and Kosik KS: A microRNA array reveals extensive regulation of microRNAs during brain development. RNA 9: 1274-1281, 2003.
44. Wilting SM, van Boerdonk RA, Henken FE, et al: Methylation-mediated silencing and tumour suppressive function of hsa-miR-124 in cervical cancer. Mol Cancer 9: 167, 2010.

45. Wang X, Tang S, Le SY, et al: Aberrant expression of oncogenic and tumor-suppressive microRNAs in cervical cancer is required for cancer cell growth. PloS one 3: e2557, 2008.

46. Lee JW, Choi CH, Choi JJ, et al: Altered MicroRNA expression in cervical carcinomas. Clin Cancer Res 14: 2535-2542, 2008.

47. van Solingen C, Seghers L, Bijkerk R, et al: Antagomir-mediated silencing of endothelial cell specific microRNA-126 impairs ischemia-induced angiogenesis. J Cell Mol Med 13: 1577-1585, 2009.

48. Wang S, Aurora AB, Johnson BA, et al: The endothelial-specific microRNA miR-126 governs vascular integrity and angiogenesis. Dev Cell 15: 261-271,2008.

49. Meister J and Schmidt MH: miR-126 and miR-126*: new players in cancer. ScientificWorldJournal 10: 2090-2100, 2010.

50. Sahasrabuddhe VV, Luhn P and Wentzensen N: Human papillomavirus and cervical cancer: biomarkers for improved prevention efforts. Future Microbiol 6: 1083-1098, 2011.

51. Liu L, Yu X, Guo X, et al: miR-143 is downregulated in cervical cancer and promotes apoptosis and inhibits tumor formation by targeting Bcl-2. Mol Med Rep 5: 753-760, 2012.

52. Wang F, Li Y, Zhou J, et al: miR-375 is down-regulated in squamous cervical cancer and inhibits cell migration and invasion via targeting transcription factor SP1. Am J Pathol 179: 2580-2588, 2011 .

53. Wei Q, Li YX, Liu M, Li X and Tang H: MiR-17-5p targets TP53INP1 and regulates cell proliferation and apoptosis of cervical cancer cells. IUBMB life 64: 697-704, 2012.

54. Pang RT, Leung CO, Ye TM, et al: MicroRNA-34a suppresses invasion through downregulation of Notch1 and Jagged1 in cervical carcinoma and choriocarcinoma cells. Carcinogenesis 31: 1037-1044, 2010.

55. Trakooljul N, Hicks JA and Liu HC: Identification of target genes and pathways associated with chicken microRNA miR-143. Anim Genet 41: 357-364, 2010.

56. Gao W, Yu Y, Cao H, Shen H, Li X, Pan S and Shu Y: Deregulated expression of miR-21, miR-143 and miR-181a in non small cell lung cancer is related to clinicopathologic characteristics or patient prognosis. Biomed Pharmacother 64: 399-408, 2010.

57. Ahmad I, Singh LB, Yang ZH, et al: Mir143 expression inversely correlates with nuclear ERK5 immunoreactivity in clinical prostate cancer. Br J Cancer 108: 149-154, 2013.

58. Deftereos G, Corrie SR, Feng Q, et al: Expression of mir-21 and mir-143 in cervical specimens ranging from histologically normal through to invasive cervical cancer. PLoS One 6: e28423, 2011.

59. Zhang H, Cai X, Wang Y, Tang H, Tong D and Ji F: microRNA-143, down-regulated in osteosarcoma, promotes apoptosis and suppresses tumorigenicity by targeting Bcl-2. Oncol Rep 24: 1363-1369, 2010

60. Slaby O, Svoboda M, Fabian P, et al: Altered expression of miR-21, miR-31, miR-143 and miR-145 is related to clinicopathologic features of colorectal cancer. Oncology 72: 397-402, 2007

61. Kitade Y and Akao Y: MicroRNAs and their therapeutic potential for human diseases: microRNAs, miR-143 and-145, function as anti-oncomirs and the application of chemically modified miR-143 as an anti-cancer drug. J Pharmacol Sci 114: 276-280, 2010. 